\title{
9. Politik, Geschichte, Recht: \\ Johann Caspar Bluntschli
}

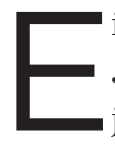

in Schweizer machte C.H.Beck seit der Jahrhundertmitte zu einem erfolgreichen juristischen Verlag, der weit über die Grenzen Bayerns hinaus wahrgenommen wurde. Die Rede ist von Johann Caspar Bluntschli. ${ }^{2}$ Als Sohn und Enkel von Schreibern der Metzgerzunft «Zum Widdern» 1808 in Zürich gebo-
Denn es werden falsche Christus und falsche Propheten aufstehen,

[...] Herr Friedrich Rohmer und Theodor Rohmer aus Zürich, und werden sagen: Hier ist Christus! Karl Marx, Die heilige Familie oder Kritik der kritischen Kritik'

ren, studierte Bluntschli zunächst in Berlin bei Friedrich Carl von Savigny und Friedrich Schleiermacher und wurde 1829 an der Universität Bonn, wo er auch Barthold Georg Niebuhr gehört hatte, mit einer römischrechtlichen Dissertation promoviert. Schon in seinem Studium fanden Recht und Geschichte zusammen. Vier Jahre später erhielt er, gerade 25 Jahre alt, einen Ruf auf eine Professur für Römisches Recht an der in seiner Heimatstadt neu gegründeten Universität. Dort war er politisch aktiv, saß mit zo Jahren im Großen Rat und wurde 1839 Regierungsrat.

\section{Friedrich Rohmer - Parteienlehre und Psychologie}

1841 machte Bluntschli die Bekanntschaft von zwei jungen Bayern, die sich zusammen mit anderen süddeutschen Intellektuellen in Zürich aufhielten: den Brüdern Friedrich und Theodor Rohmer. Durch den sogenannten Rohmerkreis fand Bluntschli zu C.H.Beck. Theodor Rohmer, 1820 geboren, hatte, gerade einmal 2o-jährig, seine erste Schrift bei C.H.Beck herausgebracht, die «Deutschlands Beruf in der Gegenwart und Zukunft» in der Überwindung konfessioneller und politischer Spaltungen, in der Herstellung nationaler Einheit, in der Abwehr demokratischer Forderungen und im Aufbau einer kontinentalen Hegemonie erblickte. ${ }^{3}$ Den Kontakt mit dem Verleger Carl Beck hatte Karl Brater hergestellt, der Theodor und seinen zwei Jahre älteren Bruder Ernst vom Münchner Gymnasium her kannte. Braters Vater war zudem Pate von Theodor. ${ }^{4}$ "Deutschlands Beruf in der Gegenwart und Zukunft» erschien 1841 auch bei Julius Fröbel, 
d.h. im Verlag des Literarischen Comptoirs in Zürich und Winterthur, und wurde von Bluntschli in dem «Beobachter aus der östlichen Schweiz» vom 13. Oktober 184,1 besprochen. Die Redaktion des katholischen und konservativen Blattes legte er in die Hände der beiden Brüder, die es für ihre Polemik gegen die «Radikalen» und «Republikaner» nutzten. ${ }^{5}$ Allgemeines Wahlrecht, plebiszitäre Elemente der Partizipation und demokratische Kontrolle der Exekutive waren ihnen wie ihrem Zürcher Förderer ein Graus. Rasch schlugen die Angegriffenen zurück und empörten sich öffentlich über die «ungezogenen Abenteurer», die wieder dort hingehen sollten, wo sie hergekommen waren. «Wir Schweizer», so schrieb ein helvetischer Zeitgenosse, «brauchen sie nicht. Am besten wäre es, wenn Herr Bluntschli mit ihnen wanderte.» ${ }^{6}$

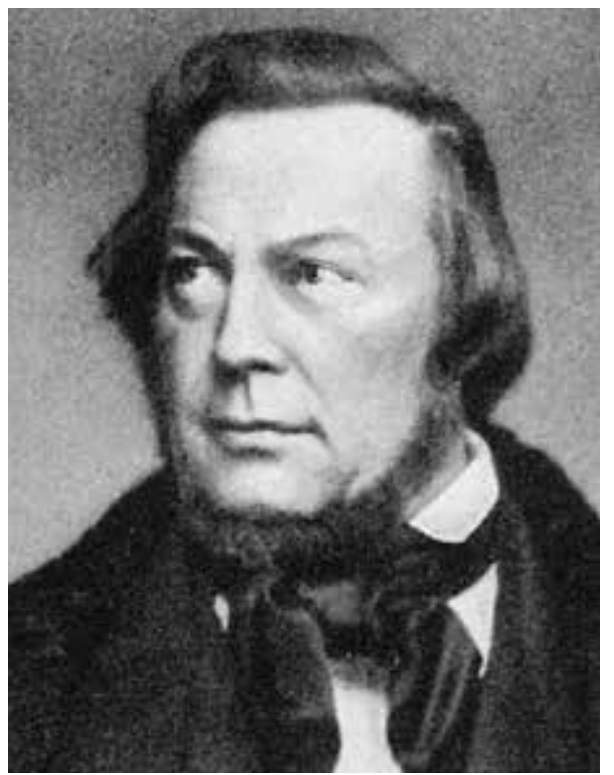

Johann Caspar Bluntschli

Zur Empörung weiter Kreise predigte der Pfarrerssohn Theodor Rohmer, die Ankunft eines «modernen Messias der Wissenschaft» sei erfolgt, der Deutschland seiner Bestimmung zuführen sollte. Dieser nationale Erlöser war in seinen Augen kein anderer als sein 27-jähriger Bruder Friedrich, der in Zürich mit Bluntschlis Applaus als eine Art politischer Guru auftrat und mit Bluntschlis Geld ein Auskommen fand. Der charismatische Heilskünder, der in München Philosophie studiert hatte, orakelte, das Recht sei die Existenz, und der Moment, in dem sich Quantität und Qualität, d. h. Volk und Herrscher, sonderten, sei der Augenblick, in dem der Staat entstehe. Im Mittelpunkt des Gedankengebäudes stand die Lehre von Potenzen der menschlichen Seele, die mit einer psychologistischen Generationentheorie politischer Repräsentation verknüpft wurde. Vier mal vier, mithin sechzehn Grundkräfte sollten den Einzelnen ebenso bestimmen wie die Politik. Seine Parteienlehre erkannte in Analogie zu den vier Lebensaltern des Menschen Kindheit, Jugend, Mannesalter und Greisenalter - vier Konstanten des politischen Lebens: die Radikalen, die Liberalen, die Konservativen und die Absolutisten. In verschiedenen historischen Epochen seien diese vier Richtungen in fester Abfolge immer wieder zur Herrschaft gelangt. Der Radikalismus wurde mit der infantilen Altersstufe verglichen, der Libera- 
lismus mit der «bewussteren Periode des jungen Mannes», der Konservativismus trug «die Züge des reiferen Mannes» und der Absolutismus zeigte «die Herrschaft der Grundkräfte, welche in dem höheren Lebensalter hervortreten und den alten Mann charakterisieren». ${ }^{7}$

Bluntschli war von Rohmer, dessen «Aufbruch zur Romantik» in einem zweifelhaften Psychologismus endete, sofort fasziniert. Ihm gefielen die Verbindung von Wissenschaft und Politik, die Rohmer einforderte, seine säkulare Heilsbotschaft, die Welt durch das «Wissen über die höchsten Dinge» erlösen und eine «neue, vernünftige und bessere Weltordnung» stiften zu wollen, und sein politischer Konservativismus, der «radikale» Ideen negierte und die monarchische Herrschaft als ideale Staatsform propagierte. ${ }^{8}$ Frühere Weggefährten waren fassungslos, dass Bluntschli Friedrich Rohmer verfiel, dem «Stifter einer fanatischen politisch-religiösen Sekte, deren in Deutschland zerstreut lebende Mitglieder teils ihn wirklich für einen politischen Messias und sich für seine Jünger hielten, teils bewusst die doppelte Gaunerei von politischen Agenten und literarischen Industrierittern mitmachten». ${ }^{9}$

Bluntschli und die Rohmer-Brüder verfolgten indes ein handfestes politisches Ziel: die Gründung einer liberal-konservativen Partei. Dazu entwickelten sie eine Parteienlehre, die 184,2 zunächst in einer Artikelserie im «Beobachter aus der östlichen Schweiz», zwei Jahre später aber von Theodor Rohmer in dem Buch «Die Lehre von den politischen Parteien» begründet wurde, das bei C.H.Beck in Nördlingen erschien. ${ }^{10}$ Wieder hatte Brater seine Verbindungen spielen lassen. Die Gedankenkonstrukte des Bluntschli-Rohmer-Kreises übertrugen Herders Vorstellung, die Menschheit spiegele in ihrer Entwicklung das Leben des Einzelnen, auf die politischen Parteien des Vormärz. Innere und äußere, männliche und weibliche Gemüts- und Geisteskräfte, aktive und passive Tätigkeitsseiten und Potenzen wurden bemüht, um die Überlegenheit eines konservativen Liberalismus zu verkünden. Es sollte der Nachweis geführt werden, dass «das liberale Prinzip» das «höhere, fruchtbarere, bewusstere, männlichere» sei, der «Radikalismus» hingegen «unreif, kindlich, erfahrungslos, knabenhaft». Die «männlichen Conservativen» wiederum erkannten, dass «sie den echten Liberalen viel» näher stünden, «als sie zuvor gedacht hatten». Die Schlussfolgerung lautete: «Die geistige Leistung kam nun den Liberalen zu, die Radikalen wurden von der geträumten Höhe gestürzt, sie mussten der Führung der Liberalen folgen.»11 Bluntschli und seine Mitstreiter wollten in den politisch-religiösen Auseinandersetzungen, die die Schweiz Anfang der 1840er Jahre in verschiedene Parteiungen spaltete, zwischen ge- 
mäßigten Liberalen und reformierten Konservativen vermitteln. Zur theoretischen Begründung einer juste-milieu-Politik berief man sich jedoch nicht auf Benjamin Constants stringente Philosophie, sondern auf Friedrich Rohmers psychologistische Doktrin. Die liberal-konservative Politik war nicht das Ergebnis persönlicher Präferenzen oder kontingenter Ereignisse, sondern durch einen vermeintlich wissenschaftlichen Ansatz quasi gesetzmäßige Notwendigkeit. Diese Agitation polarisierte den Zürcher Parteienkampf und brachte der neu gegründeten liberal-konservativen Partei bei den Maiwahlen 184,2 eine knappe Mehrheit im Großen Rat. ${ }^{12}$ Friedrich Rohmer aber, von der radikalen und liberalen Presse geprügelt, verließ die Schweiz schon 1843 wieder.

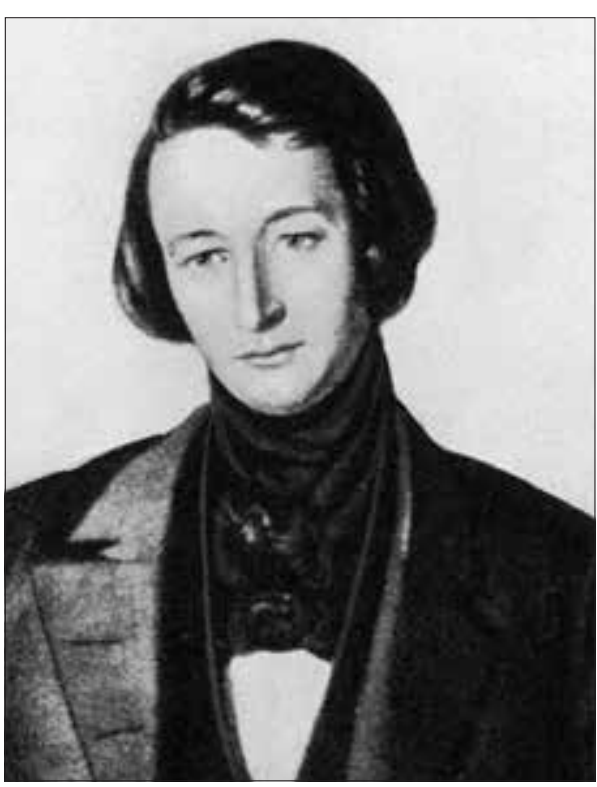

Friedrich Rohmer

Bluntschli stand zu Friedrich Rohmer. Er verbreitete die Vorstellung vom Staat als einer mit Körper und Geist ausgestatteten Person in seinen «Psychologischen Studien über Staat und Kirche», die 1844 bei C.H.Beck in Nördlingen erschienen. In dem organizistischen Modell wurde der Staat als ein lebendiger Körper verstanden. Das Verhältnis von Staat und Kirche beschrieb er in Analogie zur geschlechtsspezifischen Rollenverteilung zwischen Mann und Frau. ${ }^{13}$ Im selben Jahr scheiterte er denkbar knapp bei der Zürcher Bürgermeisterwahl von 1844. Seine Verbindung zu dem «dahergelaufenen Deutschen» hatte ihn die Mehrheit gekostet. ${ }^{14} 184,5$ erklärte er seinen Rücktritt aus der kantonalen Regierung. Nach dem Sieg der fortschrittlichen Kantone im Sonderbundkrieg von 1847 beschloss er, die Schweiz zu verlassen. In seinen Augen war seine Politik gescheitert, der Radikalismus hatte gesiegt, ein Ausgleich in der Mitte schien ihm unmöglich. ${ }^{15}$ Seine Blicke richteten sich nach München, wo Friedrich Rohmer lebte. ${ }^{16}$

Nach dem Scheitern seiner politischen Hoffnungen in der Heimat wollte Bluntschli seine ganze Kraft Deutschland widmen. Der Schweizer assistierte Rohmer und wurde zum Verteidiger der Monarchie, die aber durch notwendige Reformen gestärkt werden sollte. Ziel war eine Allianz von Liberalen und Konservativen auch in Bayern, um "gemeinsam die Extreme der Radikalen und der Absolutisten zu bändigen». ${ }^{17}$ Im März 184, 8 , mit- 
ten in den Wirren um die skandalträchtige Abdankung Ludwigs I., der infolge seiner amour fou zu der irischen Tänzerin Lola Montez auf den Thron verzichten musste, erschien Friedrich Rohmers Schrift über den vierten Stand und die Monarchie bei C.H.Beck. ${ }^{18}$ Der bayerische König sollte überzeugt werden, dass nur verfassungs- und sozialpolitische Reformen die drohende Revolution verhindern könnten. Das Bürgertum müsse «in vollen Genuss der konstitutionellen Rechte treten [...], ohne jedoch der Krone ihre Selbständigkeit, dem Adel seine Standschaft zu nehmen». Zugleich sei es «Krone, Adel und Bürgerthum» aufgetragen, «für's niedere Volk zu sorgen», auf dass der vierte Stand und der König zusammenfänden: «Die Initiative für den vierten Stand ist die Grundlage der Monarchie.» Einen Moment lang schien es, als könnte ein «soziales Königtum» an der Isar verwirklicht werden, das die «Volksinteressen» berücksichtigen sollte. Eine solche Monarchie war Bluntschli und Rohmer «lieber als die Republik». ${ }^{19}$ Die paternalistische Sozialpolitik der Krone wollte weniger die Not des Proletariats lindern als der monarchischen Herrschaft eine plebiszitäre Legitimation verschaffen. Doch der bonapartistischen Agitation des politisierenden Philosophen war kein Erfolg beschieden. Ludwig I. musste abdanken, und sein Nachfolger, Maximilian II., dachte nicht daran, das Volk als Legitimationsgrund monarchischer Herrschaft zu verstehen. ${ }^{20}$

Friedrich Rohmer kandidierte spät im Jahr 184,8 bei den Abgeordnetenwahlen für den Wahlkreis Nördlingen-Neuburg. Ihn unterstützten Karl Brater und Carl Beck. ${ }^{21}$ Bluntschli hingegen wechselte Anfang November 184,8 auf einen Lehrstuhl für deutsches Privat- und Staatsrecht an der Universität München. ${ }^{22}$ Die Gefahr eines linken Umsturzes, der auch die bürgerliche Eigentumsordnung in Frage gestellt hätte, sollte durch eine Vermittlungspolitik überwunden werden. Doch nach dem Scheitern der deutschen Revolution wurden die traditionellen Herrschaftsverhältnisse wiederhergestellt. Auch in München war Bluntschli politisch nicht erfolgreich. Er konzentrierte sich auf die Wissenschaft.

Bluntschli publizierte zunächst nicht weiter bei C.H.Beck - möglicherweise eine Folge des plötzlichen Todes von Carl Beck. Der Rechtsgelehrte ging zur Zweigniederlassung der Cotta'schen Buchhandlung in München, um 1852 seine Darstellung «Allgemeines Staatsrecht geschichtlich begründet» zu veröffentlichen, in der er den juristischen Ansatz des Heidelberger Staatswissenschaftlers Robert von Mohl mit der historischen Perspektive Friedrich Christoph Dahlmanns zu einem dialektischen Konzept der Lehre vom Staat synthetisierte. Der dritte Teil der 1875 erschienenen fünften Auflage war programmatisch mit «Politik als Wissenschaft» über- 
schrieben. Ernst Rohmer versuchte in den 1870er Jahren, das Werk zu verlegen, scheiterte aber an Cottas Intervention. Man war nicht gewillt, das Verlagsrecht an Rohmer abzutreten. ${ }^{25}$ Bluntschlis dreibändige «Lehre vom modernen Staat» wurde 1875/76 von Cotta veröffentlicht. ${ }^{24}$ Die Aufgabe der Politik war die Leitung des Staates - in praktischer und theoretischer Hinsicht. Politik konnte sich folglich auf die Regierungspraxis von Akteuren beziehen, aber auch auf die wissenschaftliche Durchdringung des Gegenstands, wie sie von Professoren als «Staatsgelehrten» zu leisten war. Beide Sphären, Staatspraxis und Staatswissenschaften, durften nicht voneinander geschieden werden, sondern bedingten einander. Das Buch erlebte mehrere Auflagen und wurde von Bluntschli «als reifste Frucht» seines «langen der politischen Wissenschaft und Praxis gewidmeten Lebens» bezeichnet. ${ }^{25}$ Tatsächlich war das Werk ein großer Erfolg, der auch Bluntschlis internationale Reputation begründete.

Doch Mitte der 185oer Jahre führten persönliche Verbindungen Bluntschli wieder zu C.H.Beck nach Nördlingen. Denn dort hatte inzwischen mit Ernst Rohmer ein Bruder von Theodor und Friedrich das Sagen, die beide 1856 gestorben waren. Ernst Rohmer war $185_{1}$ auf Karl Braters Empfehlung bei C.H.Beck verpflichtet worden, zur Unterstützung Carl Becks, der ein Jahr später unerwartet starb. Der am 29. Dezember 1818 geborene Buchhändler Rohmer schaute auf ein wechselvolles Leben zurück: Nach dem Tod seines Vaters wuchs er zusammen mit seinem jüngeren Bruder Theodor in der Familie des Konsistorialrats und Schulreformers Friedrich von Niethammer in München auf. Die Mutter, die aus der weitverzweigten württembergischen Gelehrtenfamilie Planck stammte, hatte ihre Verbindungen spielen lassen, so dass ihre beiden Söhne im Geiste des Humanismus und in der Gewissheit des Luthertums aufwuchsen. Ernst Rohmer absolvierte zunächst eine kaufmännische Ausbildung. Da weder die familiären Mittel noch die wissenschaftlichen Gaben für ein Studium reichten, wandte er sich dann dem Buchhandel zu und arbeitete seit 1840 als Gehilfe in Ulm, Freiburg im Breisgau, Koblenz, Stuttgart und Landau. Die Revolution von 184,8 ließ ihn ins Journalistenfach wechseln; erst schrieb er für die «Süddeutsche politische Zeitung» in Stuttgart, dann berichtete er für die «Augsburgische Abendzeitung» aus dem Landtag und arbeitete schließlich für den «Nürnberger Korrespondenten». Energisch vertrat er die Position derer, die für eine konstitutionelle Monarchie eintraten. Zu weit gehende demokratische Forderungen waren ihm ein Graus. Die Badische Revolution verurteilte er als «die vollkommenste Anarchie», und in Gustav Struve und Friedrich Hecker sah er eine Gefahr für alle an- 
ständigen Bürger. In der bayerischen Pfalz sollten militärische «Befehlshaber mit ausgedehnten Vollmachten» für Ordnung sorgen. ${ }^{26}$

Dann stürzte ihn das Scheitern der Revolution in eine tiefe Krise. Der Sieg der Reaktion «verleidete ihm die journalistische Tätigkeit», wie Oskar Beck schrieb. Rohmer entschloss sich im Juli 1851, in den Nördlinger Verlag einzutreten, um der Schreibstube entfliehen zu können. Erst arbeitete er für Carl Beck, dann für Eugenie und Wilhelm Beck. Rasch richtete er sich in der Kleinstadt ein, holte seine Schwester, die Witwe des Malers Alexander Bruckmann, aus Stuttgart mit ihren drei Kindern zu sich und gründete mit ihnen und einer weiteren unverheirateten Schwester einen Haus-

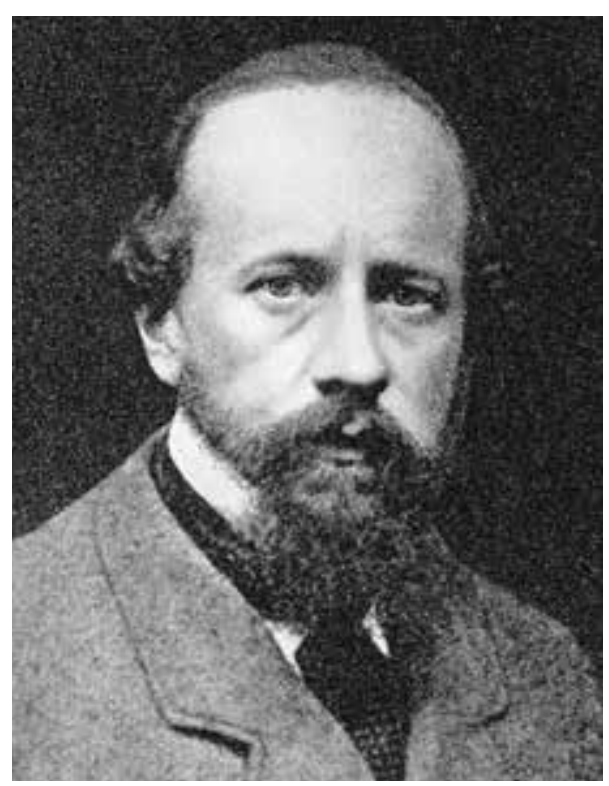

Wilhelm Beck, der kein Verleger sein wollte stand. Rohmer wurde rasch zur tragenden Säule des Geschäfts, und am ı. Juni 1857 heiratete er, nach dreijährigem Werben, Eugenie Beck, die, seinen eigenen Worten zufolge, eine gescheite und religiöse Frau war. C.H.Beck hatte einen neuen Verleger. Sein Schwager Wilhelm überließ Ernst Rohmer die Geschäftsführung des inzwischen bald 10o-jährigen Unternehmens. ${ }^{27}$ Am 2. Mai 1858 kam ein Sohn zur Welt, der nach den beiden verstorbenen Brüdern Friedrich Theodor Georg Eugen genannt wurde; gerufen werden sollte er Eugen, mit dem Namen, «den Fritz und Theodor sehr liebten». ${ }^{28}$

\section{Zwei Nachlassverwalter}

Nichts beschäftigte Ernst Rohmer und Johann Caspar Bluntschli intensiver als die Bemühungen, die Schriften Friedrich Rohmers herauszugeben und eine umfassende Biographie folgen zu lassen. Ihr intensiver Briefwechsel kreist immer wieder um dieses Thema. Am 16. Juli $185^{2}$ hatte Bluntschli Friedrich und Theodor Rohmer ein Darlehen von 1000 Gulden gewährt, zu einem Zinssatz von vier Prozent. Als Sicherheit wurden die Schriften Friedrich Rohmers hinterlegt. Die Beilage zu dem Darlehensvertrag bot die Werkübersicht und antizipierte die geplante Ausgabe. ${ }^{29}$ Bluntschli half 
bei der Neuauflage von Friedrich Rohmers «Kritik des Gottesbegriffes in den gegenwärtigen Weltansichten» $(1856)^{30}$ und der postumen Edition von «Gott und seine Schöpfung» (1857). ${ }^{31}$ Beide Werke erschienen anonym; die Vermeidung des immer noch umstrittenen Autorennamens sollte die Verbreitung der Schriften befördern. 1858 folgte dann «Der natürliche Weg des Menschen zu Gott».

Johann Caspar Bluntschli stellte in seinen Erinnerungen fest, dass man ihn als Lehrer des Staatsrechts schätze. Auf diesem Feld habe er sich einen Namen erworben, der auch im Auslande bekannt sei. Aber als seine bedeutendste Leistung erachtete er, dass er «Friedrich Rohmer und seine Lehre verstanden habe». ${ }^{32}$ Schon nach Friedrich Rohmers frühem Tod am 1. November 1856 hatte der Schweizer «im Angesicht der Leiche» gelobt, «sein Vermächtnis an die Welt [...] zu vollziehen». Seine Aufgabe sah er darin, zusammen mit den wenigen Getreuen «die Samenkörner der Wissenschaft noch in die Erde zu legen», damit sie «dereinst aufgehen» möchten. Seine Hoffnung, zukünftige Generationen würden erkennen, dass von Rohmers «Ideen aus die Heilung kommen wird für die verwirrte Menschheit», erwies sich jedoch als Illusion. ${ }^{33}$

Am 19. Januar 1857 bat Ernst Rohmer den Münchner Professor, dieser möge ihn unterstützen, sein Lebenswerk zu realisieren, «die buchhändlerische Vermittlung der Herausgabe der Werke meiner Brüder».34 Am 28. April 1857 unterzeichnete man den Vertrag «über den Gesamtverlag der Rohmerschen Schriften». Der Reingewinn sollte zwischen der Familie Rohmer (10\%), dem Herausgeber (45\%) und dem Verlag (45\%) geteilt werden. Vorgesehen war eine Vertragslaufzeit von fünfzehn Jahren. ${ }^{35} 1858$ unterstützte Ernst Rohmer die postume Edition von Theodor Rohmers Buch über «Die Religion Jesu» und musste Auskunft geben, ob eine lange Fußnote zur christlichen Sexualmoral als «persönliche Apologie» von Friedrich Rohmers «Sinnlichkeit außerhalb der Monogamie» missverstanden werden könnte. ${ }^{36}$ Anfang 1859 ordnete er das Familienarchiv und übersandte dem «hochverehrten Freund» Johann Caspar Bluntschli die «wichtigsten Manuskripte mit den psychologischen Studien, Vieles für die Biographie Unentbehrliche, eine Anzahl der verschiedensten Reflexionen und Ideen von höchstem Interesse, aber alles bunt durcheinander». ${ }^{37}$ Bluntschli war bereits zu diesem Zeitpunkt als der Hauptverantwortliche für das Editionsunternehmen auserkoren, das die Freunde aus Zürcher Tagen, Heinrich Hottinger, Heinrich Schultheß und Gustav Widenmann, unterstützten. Später trat Rudolf Seyerlen hinzu, ein Schüler von Friedrich Chr. Baur in Tübingen, der 1875 auf den Lehrstuhl für Praktische Theolo- 
gie in Jena berufen wurde. Bluntschli übernahm die Herausgabe der «Wissenschaft von Gott» und die Biographie Friedrich Rohmers. ${ }^{38}$

1860 erschien die erste nicht auf Friedrich Rohmer bezogene Publikation Bluntschlis im Verlag C.H.Beck, die «Meinungsäußerung eines Publizisten über die neueren Concordate» im Umfang von $z_{1}$ Seiten. Als sich der Münchner Ordinarius im folgenden Jahr anschickte, an die Universität Heidelberg zu wechseln, bekundete Rohmer nicht nur, dass es durch dessen Weggang «mit dem Glanze der Universität München so ziemlich vorbei sein» werde, sondern gestand, dass er «zu gerne ein größeres Werk» von Bluntschli in seinen Verlag «bekommen» möchte und bereit sei, «jede Konkurrenz zu bestehen».39 Doch die «Geschichte des Allgemeinen Staatsrechts und der Politik», auf die Rohmer geschielt hatte, erschien 1864 ebenfalls bei der Cotta'schen Buchhandlung. ${ }^{40}$ C.H.Beck musste sich zunächst mit der umfangreichen Sammlung der deutschen Rechtssprichwörter begnügen, an der Bluntschli mitgearbeitet hatte.

1866 veröffentlichte Rohmer dann als erste eigenständige Monographie «Altasiatische Gottes- und Weltideen in ihren Wirkungen auf das Gemeinleben der Menschen». Es handelte sich um fünf Vorträge, die Bluntschli im Winter 1864/65 in Karlsruhe gehalten hatte. Die Gelegenheitsschrift, von der gerade einmal 100 Exemplare gedruckt wurden, erschien nur deshalb bei C.H.Beck, weil Rohmer den renommierten Wissenschaftler Bluntschli auch als juristischen Autor gewinnen wollte. Rohmer hatte schon Ende Dezember 1864, bekannt: «Die Aussicht, Ihre Vorlesungen in Verlag zu bekommen, ist mir außerordentlich erfreulich.» ${ }^{41}$ Ein gutes Jahr später hoffte er auf Bluntschlis völkerrechtliche Studien. ${ }^{42}$ In der Tat konnte C.H.Beck 1866 «Das moderne Kriegsrecht der civilisierten Staaten» herausbringen; dieses Mal betrug die Auflage gut 1000 Exemplare. ${ }^{43}$ Als sich in den ersten Maitagen abzeichnete, dass die preußisch-österreichischen Auseinandersetzungen militärisch zu eskalieren drohten, bedauerte Rohmer als Geschäftsmann die niedrige Auflage. «Ihr 〈Kriegsrecht〉 ist nun allgemein versendet und scheint leider Gottes! zu rechter Zeit zu kommen. Es ist ärgerlich, dass die Auflage aus $105^{\circ}$ Exemplaren gemacht wurde, da ich nur sehr sparsam versenden konnte. Aber wer wollte annehmen, dass es wirklich so weit käme!» ${ }^{44}$ Doch im Juni, mitten in den Kampfhandlungen, ventilierten Autor und Verleger die Möglichkeit, «eine Volksausgabe des Kriegsrechtes» zu veranstalten. Die Auflage sollte 50oo Exemplare stark werden, das Exemplar 18 Kreuzer kosten und der Satz stehen bleiben. Rohmer hoffte, dass der preußische Generalstab eine Sammelbestellung in Auftrag geben würde, und wollte Exemplare an alle Kriegsministerien 
senden. ${ }^{45}$ Im Frühjahr 1867 regte Rohmer eine Neuauflage des «Kriegsrechtes» an, in welcher der Autor die Erfahrung des Deutschen Krieges von 1866 aufgreifen «und das Ganze vielleicht in einer mehr populären Form» darbieten sollte. Der Geschäftsmann Rohmer spekulierte in der Luxemburgkrise, als der intendierte Kauf des Großherzogtums Luxemburg durch Napoleon III. auf der internationalen Bühne für Unruhe sorgte, dass dann «in den Hansestädten lebhafte Nachfrage damit erzielt werden könnte, auch vielleicht bei uns und am Rhein». ${ }^{46}$ Diese Ausgabe ließ sich allerdings ebenso wenig realisieren wie eine französische Übersetzung. Der Absatz der ersten Auflage war doch schleppender als ursprünglich gedacht. Allerdings erschien 1874 eine «Separatausgabe» mit einer Auflage von 1500 Exemplaren. Mehr wollte Rohmer nicht wagen. Bluntschli erhielt ein Honorar von 100 Gulden, musste aber kürzen, damit «das Gesamtvolumen wenigstens auf 8 Bogen reduziert werden» konnte. ${ }^{47}$

\section{Bluntschli - der Staats- und Völkerrechtler}

C.H.Beck war durch Friedrich Rohmer zu Bluntschlis Hausverlag geworden. Am 15. November 1868 schrieb Ernst Rohmer an seinen Autor: «Mit Freuden habe ich ersehen, dass Sie mit Ihrer Schrift über den Charakter und Geist der politischen Parteien schon fertig sind. Natürlich rechne ich darauf, dass Sie mir den Verlag anvertrauen.» ${ }^{48}$ Die Schrift verbreitete die Parteienlehre Friedrich Rohmers und war deshalb in Nördlingen hochwillkommen. Rezipiert wurde sie nicht. Heinrich von Treitschke wies sie in den «Preußischen Jahrbüchern» von 1871 zurück. Bluntschli verteidigte seine - und damit Rohmers - «psychologische Parteienlehre» in einem langen Brief an den Historiker, der sich unbeeindruckt zeigte. ${ }^{49}$

Einflussreicher als diese Schrift waren für den öffentlichen Diskurs Bluntschlis Überlegungen zu einem «modernen Völkerrecht der zivilisierten Staaten», die 1868 zum ersten Mal bei C.H.Beck erschienen. Bluntschli strebte vor allem nach der Kodifizierung eines Kriegsvölkerrechts, das einen Krieg dann und nur dann als gerecht anerkennt, «wenn und soweit die bewaffnete Selbsthilfe durch das Völkerrecht begründet ist». Dann argumentierte er stringent für die Anwendung des ius in bello. Der «Völkerrechtsklassiker» vermittelte angloamerikanische Positionen der Völkerrechtslehre des 19. Jahrhunderts und beeinflusste die juristische Diskussion nachhaltig. ${ }^{50}$ 1881 wurde es auf Veranlassung der chinesischen 
Regierung sogar ins Englische übersetzt. Ernst Rohmer kommentierte: «Das ist ja eine Anerkennung, die etwas heißen will!»51

Anfang des Jahres 1872 kam es jedoch zu einer Verstimmung zwischen dem Verleger und seinem gelehrten Autor. Zum Jahreswechsel hatte Rohmer dem Freund noch «den innigsten Dank» abgestattet «für die tiefe Pietät und Treue, welche Sie der Wissenschaft und dem Andenken Fritz's und des ihm innig verbundenen Theodor's bewahrt haben und mit der Sie im ablaufenden Jahre ein so großes Stück dieser schweren Denkarbeit für die Nachwelt gerettet haben!»52 Dann traf der Teil von Bluntschlis Biographie ein, in der er Friedrich Rohmers Leben bis 184,2 behandelte. Ernst Rohmer kritisierte, dass Bluntschli nicht «im nötigen Maße» das «Anstößige» entfernt habe. ${ }^{53}$ Die Lektüre sei abstoßend, und Friedrich Rohmer werde ohne Zutun seiner Gegner «als Schwindler und eine sittlich sehr problematische Natur» dargestellt. Die Schlussfolgerung war eindeutig: Diese Lebensbeschreibung sollte nicht erscheinen. «Ich hoffe», schrieb Rohmer an Bluntschli, «Sie entschließen sich dazu, die Biographie einer späteren Zeit vorzubehalten, wenigstens in der vorliegenden Form.» ${ }^{54}$ Bereits sechs Jahre zuvor hatte Rohmer auf einem Treffen in Konstanz darauf bestanden, dass die Biographie das Leben nur skizziere und sich ansonsten auf die «Entwicklung der Wissenschaft» konzentriere. ${ }^{55}$ Alkoholabusus und Aggressivität, Unzuverlässigkeit und Unbeherrschtheit, Wutausbrüche und Widerwärtigkeiten sollten nicht Gegenstand einer Lebensbeschreibung sein, die vorzugsweise das verkannte Genie zu porträtieren hatte. Die eindeutigen Vorgaben sorgten im Herausgeberkreis immer wieder für Spannungen. ${ }^{56}$ Auch noch nach Bluntschlis Tod im Jahre 1881 versuchte Ernst Rohmer die Veröffentlichungen über seinen Bruder zu zensieren, indem er Bluntschlis Erben mitteilte, dass dieser die Biographie «zunächst für sich und die Seinigen niedergeschrieben» habe und dass sie daher «nicht gedruckt werden sollte». ${ }^{57} \mathrm{Um}$ sichergehen zu können, dass er die Kontrolle über Bluntschlis Arbeiten behielt, kaufte Rohmer von den Erben die Verlagsrechte an Bluntschlis Werken und das Manuskript der Friedrich-Rohmer-Biographie. Auch im Manuskript von Bluntschlis Autobiographie, das Rohmer für 4500 Mark erwarb, mussten Streichungen vorgenommen werden. ${ }^{58}$ Der Verleger agierte als Zensor, und sein Erfüllungsgehilfe war Rudolf Seyerlen, der die «Denkwürdigkeiten aus meinem Leben» nicht nur postum veröffentlichte, sondern vorher auch «gereinigt hatte. ${ }^{59}$

Bluntschlis staatswissenschaftliche Publikationen waren Rohmer hingegen höchst willkommen. «Mit Vergnügen ersehe ich», schrieb der Ver- 
leger am 3. November 1873, «dass Sie den Plan der Herausgabe einer populären Staatslehre der Verwirklichung entgegenzuführen im Begriffe sind.» ${ }^{60}$ Die «Deutsche Staatslehre für Gebildete», die im folgenden Jahr erschien, fasste Bluntschlis staatsrechtliche und -wissenschaftliche Arbeiten zusammen und erreichte $\mathbf{1 8 8 0}$ in zweiter Auflage unter dem Titel «Deutsche Staatslehre und die heutige Staatenwelt» die Leser. Rohmer hatte als Titel «Staatslehre für die Deutschen» vorgeschlagen, der «einem stolzen Nationalgefühl Rechnung» trage. ${ }^{61}$ Der Schweizer Jurist, der sich der deutschen Sache verschrieben hatte, stimmte jedoch dem Vorschlag nicht zu. In der Schrift widmete er dem «zusammengesetzten Staat» ein Kapitel, den er in der Tradition der griechischen Verfassungstheorie durch eine Mischverfassung charakterisiert sah. Diese alte res publica mixta hat in der jüngsten Vergangenheit eine Renaissance erfahren; allerdings spricht die aktuelle Forschung heute von composite state oder composite monarchy. Bluntschli sah die höchste Form der Staatlichkeit nicht in einem national homogenisierten Staat, sondern vielmehr in dem Zusammenleben verschiedener Nationen in einem Staat. Der Schweizer Jurist interpretierte die confoederatio Helvetica als Zusammenschluss von Kantonen, die er als durchweg «nationale Staaten» verstand; die Schweiz war damit ein Vorbild für einen zusammengesetzten Staat. ${ }^{62}$ Man hat dem Staatswissenschaftler zu Recht Widersprüchlichkeiten und Inkonsistenzen vorgehalten, fehlende juristische Präzision und eine unklare Begrifflichkeit; ${ }^{63}$ dennoch war seine Staatslehre für die verfassungstheoretischen Debatten in Deutschland einflussreich. Das Repräsentationssystem, das Bluntschli verfocht, lehnte das Publikum zunächst ab, weil man glaubte, es stehe in Widerspruch zum monarchischen Prinzip; aber durch die von den Verlagen C.H.Beck und Cotta verbreiteten Schriften Bluntschlis wurde die Lehre von der Repräsentation zum Ausgangspunkt verfassungsrechtlicher Entwürfe einer konstitutionellen Monarchie im Kaiserreich. ${ }^{64}$ Der Schweizer Gelehrte und Politiker vertrat rechtsliberale Positionen, favorisierte die deutsche Einigung von oben statt von unten und vertraute auf den «königlich preußischen Revolutionär» Bismarck. ${ }^{65}$

Bluntschli begründete vor allem Politik als Wissenschaft. Fruchtbar verband er juristische und historische Ansätze, und energisch plädierte er für die Zusammenarbeit von Theorie und Praxis in der Staatswissenschaft. ${ }^{6}$ Deren akademische und öffentliche Wahrnehmung förderte er durch das zusammen mit Karl Brater herausgegebene «Deutsche Staats-Wörterbuch», das zwischen 1857 und 1870 auf elf Bände wuchs und den bisherigen Klassiker, das «Rotteck-Welckersche Staatslexikon», ersetzte. Zu den 
Autoren zählte die wissenschaftliche Prominenz, zählten insbesondere Juristen, Nationalökonomen und Historiker: Robert von Mohl, Wilhelm Roscher, Albert Schäffle, Wilhelm Stahl, Heinrich von Sybel und Heinrich von Treitschke.

1861 hatte Bluntschli München verlassen, als ihm ein Sitz in der Ersten Kammer des badischen Landtags angeboten wurde. Er wechselte auf einen Lehrstuhl für Staatswissenschaften an der Universität Heidelberg. Fortan konnte er Wissenschaft und Politik verbinden. Der Gegner des Ultramontanismus war ein engagierter Freimaurer, Präsident der badischen evangelischen Generalsynode und Mitglied im liberalen «Deutschen Protestantenverein», der 1865 in Eisenach gegen die kirchliche Reaktion gegründet worden war. Er erkannte klar die politische Resignation der deutschen Gebildeten, die nach 184,8 um sich gegriffen hatte, und kritisierte das Fehlen realpolitischer Lösungen. Als preußische Truppen im Sommer 1866 den Krieg eröffneten, schrieb er an einen befreundeten Schweizer Liberalen: «Sie haben keine Vorstellung davon, in welchem Grade die Deutschen unpolitisch sind. Sie treiben Juristerei, kritisieren über Alles und Jedes, sind voll von moralischen Erwägungen; aber vom Staat haben sie noch keine Vorstellung, und die Politik ist ihnen im Herzensgrunde verhasst, weil die liebe «Gemütlichkeit〉 bei Bier und Sang dabei aufhört.» Die deutsche Nation müsse zum Staate erzogen werden. Aber «nur die harte Schule zunächst des Krieges, dann der Taten, wird allmählich Änderung bewirken und neue Generationen bilden». ${ }^{67}$

Als Anfang September 1866 der Streit um Bismarcks Indemnitätsvorlage, die die preußischen Staatshaushalte seit 1862 nachträglich billigte und den Verfassungskonflikt in Preußen beendete, die Deutsche Fortschrittspartei spaltete, gehörte Bluntschli, der damals im badischen Heidelberg lehrte, zu den zunächst noch wenigen preußenfreundlichen Liberalen in Süddeutschland, die die Gründung der Nationalliberalen Partei und die Berliner Politik vorbehaltlos unterstützten. Der Schweizer brachte es auf den Punkt: «Aber es handelt sich schon lange nicht mehr um den Grafen Bismarck, sondern um den preußischen Staat, der den Grafen Bismarck überleben wird. Es ist nun einmal unbestreitbar, dass die Neugestaltung von Deutschland nur mit Preußen möglich ist, ohne Preußen niemals.» ${ }^{68}$ Die Ambivalenzen der Bismarck'schen Politik und seines elaborierten Herrschaftssystems erkannte Bluntschli jedoch klar. Er bezeichnete den Kanzler als einen «antediluvianischen Mann», in dem «eine seltsame Verbindung von lauterster Offenheit und tiefer Verschlagenheit, von rückhaltloser Wahrhaftigkeit und bewusster Täuschung» zu finden sei. ${ }^{69}$ Seine Agi- 
tation für die kleindeutsche Lösung brachte ihn Ende April 1868 direkt zu Bismarck, der ihn zu einer längeren Besprechung über die deutsche Einheit in Berlin empfing. ${ }^{70}$

\section{Wider das Vergessen}

1892 war die zwanzig Jahre zuvor begonnene Veröffentlichung von Friedrich Rohmers «Wissenschaft und Leben» abgeschlossen. Die ersten vier Bände enthielten die philosophischen (Bd. I-III) und die politischen Schriften (Bd. IV); die beiden letzten seine Biographie (Bd. V-VI). Das Ziel des Unternehmens war die «Ehrenrettung» Friedrich Rohmers, der angeblich «Vom Parteihass verfolgt» worden sei, so dass «eine objective Beurteilung» seines Werkes und Lebens ausstehe. ${ }^{71}$ Mit Erstaunen nimmt der Leser zur Kenntnis, dass Friedrich Rohmer sich «auf die Dauer» keineswegs «für einen Messias» gehalten habe, «wohl aber für ein welthistorisches Genie», für einen «Religionsstifter» und «Weltherrscher». ${ }^{72}$ Rohmers Selbstwahrnehmung als «phlegmatisch-cholerisches Langhorn-Genie», «in welchem die Denkkraft im höchsten Maße ausgesprochen» sei, entsprach nicht der Fremdwahrnehmung als notorischer Querulant, der erst in ein Weinhaus einkehrte, um dann durch Gewalttätigkeiten alle aus seiner Umgebung zu verscheuchen. ${ }^{73}$ Verlegerisch war das Projekt ein kompletter Misserfolg. Die Bände erwiesen sich als Ladenhüter. Ernst Rohmer musste aus «Pietät» gegenüber seinem Bruder «bedeutende materielle Opfer» bringen. Von dem ersten Band, der 1871 erschienen war, waren zehn Jahre später erst 130 Exemplare verkauft. ${ }^{74}$

Man fragt sich, weshalb Johann Caspar Bluntschli die Erinnerung an Friedrich Rohmer wachhielt. Öffentlich brachte es ihm Hohn und Spott ein. Hugo Preuß hieß Bluntschli den «wissenschaftlichen Apostel» der «mystisch-symbolischen Lehre des sonderbaren Propheten Friedrich Rohmer». ${ }^{75}$ Der «mystische Hang» gebe die einzige Erklärung für den «schier wunderbaren Einfluss, welchen Friedrich Rohmers Person und Lehre auf den sonst so verständigen, nüchtern kritischen Staatsgelehrten» ausgeübt habe. «Bluntschlis Geist blieb gefangen in diesem mystischen Irrgarten, und im Stile des Meisters prophezeite er die Herrschaft der Rohmerschen Wissenschaft genau für das Jahr 1940.»76

Wollte Bluntschli Friedrich Rohmer «historische Gerechtigkeit» zukommen lassen? ${ }^{77}$ Oder respektierte der loyale Freund die Pietät, mit der Ernst Rohmer seinem Bruder begegnete? Bluntschli fesselte, ebenso wie Ernst 
Rohmer, weniger «die philosophische Spekulation» Friedrich Rohmers als dessen «politische Ideen», wie schon Oskar Beck zu Recht hervorgehoben hat. ${ }^{78}$ Bluntschlis politische Überzeugungen oszillierten zwischen Konservativismus und Liberalismus, die konstitutionelle Monarchie feierte er als «Krone der Staatsformen»; ${ }^{79}$ Revolutionen verabscheute er, gesellschaftliche Veränderungen wollte er durch Reformen erreichen. Er verfügte nicht über die intellektuellen Mittel, sich von den bizarren Spekulationen Friedrich Rohmers zu emanzipieren. Bluntschli verstand sich zwar auf juristische Systematik und historische Kausalitäten, nicht aber auf Wissenschaftstheorie und Epistemologie.

Friedrich Rohmer selbst hat wenig zu Papier gebracht. Er war ein Meister der kleinen Form. Seine Ideen wären in Vergessenheit geraten, hätte nicht sein Bruder Theodor 1844 «Die Lehre von den politischen Parteien» bei C.H.Beck herausgegeben. ${ }^{80}$ Nicht minder wichtig war Bluntschlis Rekurs auf die Rohmer'sche Parteienlehre in Wort und Schrift. In seinem 1844 bei C.H.Beck veröffentlichten Buch über «Psychologische Studien über Staat und Kirche», in einschlägigen Artikeln im «Deutschen StaatsWörterbuch», in seiner in Nördlingen 1869 verlegten Untersuchung über «Charakter und Geist der politischen Parteien» und schließlich in seiner Studie über «Politik als Wissenschaft» verbreitete er nicht nur die Parteienlehre, sondern auch die organizistische Vorstellung vom Staatskörper. ${ }^{81}$ Mit anderen Worten: Die frohe Botschaft des selbsternannten Messias Friedrich Rohmer brachte erst sein Paulus: Johann Caspar Bluntschli in die weite Welt. Ernst Rohmer wiederum stellte den Verlag C.H.Beck zur Verfügung, um Friedrich Rohmers bizarre Lehren einer liberal-konservativen Politik publizistisch zu verbreiten. Der Erfolg war zweifelhaft. Dieser Beitrag Bayerns zur deutschen Geistesgeschichte blieb von begrenzter Wirkung. 
https://doi.org/10.17104/9783406654015-153, am 26.04.2023, 06:15:50

Open Access - (c) EY EY - http://www.beck-elibrary.de/agb 